\title{
银促进的磷自由基与异腈的加成环化反应
}

\author{
杨 斌 $a$ 田秋萍 $a$ 杨尚东*,a \\ $\left({ }^{a}\right.$ 兰州大学功能有机分子国家重点实验室 兰州 730000)
}

\begin{abstract}
摘要 以银盐为氧化剂, 简单高效地实现了磷自由基对 2-异氭基-1,1'-联苯类底物的加成环化反应. 该反应原子经济性 高、反应体系温和、底物适用范围广、产率高. 为 $\mathrm{C}-\mathrm{P}$ 键的构筑提供了一种新方法, 并且合成了一系列 6 位磷酰化菲 啶类的 N-P 配体化合物.
\end{abstract}

关键词 硝酸银; 磷自由基; 异腈; C-P 键

\section{Silver-Promoted P-radical Cyclization Reaction with the Addi- tion to Isonitrile}

\author{
Yang, $\operatorname{Bin}^{a} \quad$ Tian, $^{\text {Qiuping }}{ }^{a} \quad$ Yang, Shangdong ${ }^{*, a}$ \\ ( ${ }^{a}$ State Key Laboratory of Applied Organic Chemistry, Lanzhou University, Lanzhou 730000)
}

\begin{abstract}
A simple and efficient method for the cyclization of the addition of P-radical to 2-isocyano-1,1'-biphenyl by employing silver salt as an oxidant was developed. The high atom economy, mild reaction system and a wide range of substrates with high yields make the protocol very practical. It provides a new route for $\mathrm{C}-\mathrm{P}$ bond formation. A series of 6-phosphorylated phenanthridines were synthesized.

Keywords $\mathrm{AgNO}_{3}$; P-radical; isonitrile; $\mathrm{C}-\mathrm{P}$ bond
\end{abstract}

含磷有机分子作为一类重要的有机化合物被广泛 应用于有机合成化学、化学生物学、药物化学及材料化 学等多种领域中. 同时, 有机磷配体作为一类重要的配 体, 在金属催化的有机合成化学与配位化学中也起到了 至关重要的作用 ${ }^{[1]}$. 因此, 多年来对于含磷化合物的合 成研究非常活跃. 在过去的几年中, 我们课题组就一直 致力于研究构建 $\mathrm{C}-\mathrm{P}$ 键的新方法并取得了一些进展 ${ }^{[2]}$.

到目前为止，已经发展了很多构建 $\mathrm{C}-\mathrm{P}$ 键的方法, 其中包括磷试剂与有机金属试剂的亲电取代反应及过 渡金属催化的偶联反应等 ${ }^{[3 \sim 7]}$. 氧化产生磷自由基对不 饱和键加成继而构筑 C-P 键的方法也一直受到化学家 们的关注, 很多课题组包括我们小组之前也对此方法有 过一定的探究 ${ }^{[8,2 c]}$. 虽然构筑 $\mathrm{C}-\mathrm{P}$ 键的方法已经有了很 多报道, 但是如何更为简单高效地合成含磷化合物依旧 值得化学家去进一步探索.

最近, 在我们前期研究的基础上 ${ }^{[2 c]}$, 我们又发展了 一种通过银盐氧化二苯基磷氧产生磷自由基对异腈的 加成环化反应(Eq. 1). 结果表明, 该反应体系简单、原子
经济性高, 并能在相对温和条件下通过一步反应得到 6 位磷酰化的菲啶，为合成此类 N-P 配体提供了一种新的 途径. 与此同时, 就在我们的工作已经完成正在准备稿 件的时候, 德国的 Studer 小组 ${ }^{[9]}$ 发表了与我们相似的工 作. 相比较他们的工作, 我们的反应条件更温和, 银盐 的用量少, 得到产物的产率普遍较高, 且杂环底物同样 能够兼容.

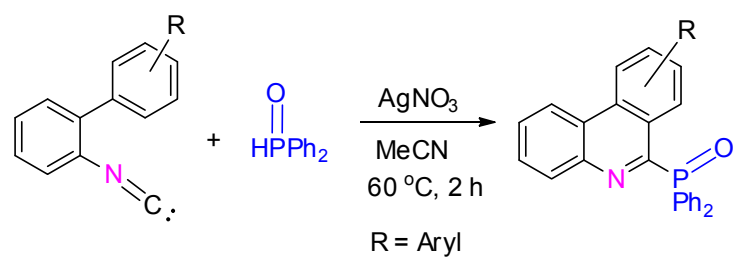

\section{1 结果与讨论}

\section{1 反应的条件优化}

我们以 2 -异氧基-1,1'-联苯(1a)和二苯基磷氧(2)为 模板底物、 2 equiv.的 $\mathrm{AgNO}_{3}$ 为氧化剂、 $2 \mathrm{~mL}$ 乙腈为溶

\footnotetext{
*E-mail: yangshd@1zu.edu.cn

Received December 18, 2013; revised January 2, 2014; published online January 3, 2014

Project supported by the National Natural Science Foundation of China (No. 21272100).

国家自然科学基金(No. 21272100)资助项目.
} 
剂在 $30{ }^{\circ} \mathrm{C}$ 下反应 $2 \mathrm{~h}$ 以 $40 \%$ 的分离产率得到目标产物 3a(表 1, Entry 1). 3a 的结构已经通过单晶证实(CCDC: 976679). 我们首先篎选了不同的银盐, 发现只有当 $\mathrm{AgNO}_{3}$ 作氧化剂才能得到较高产率(表 1, Entries 2, 3). 当使用 2 equiv. 的 $\mathrm{AgNO}_{3}$ 时产率有一定的提高(表 1, Entry 4). 在对反应温度的篎选中发现, 随着温度的升 高, 产率也随之增加, 当温度到达 $60{ }^{\circ} \mathrm{C}$ 后产率没有显 著的变化(表 1, Entries 5，6). 对溶剂的篎选表明(表 1, Entries 7９), 乙腈依然是最优选择. 当 2-异氧基-1,1'联苯和二苯基磷氧的物质的量的比调整到 $1: 1.5$ 时, 我 们能以 $86 \%$ 的产率得到 $3 \mathbf{a}$. 基于文献的报道 ${ }^{[10]}$ 和之前

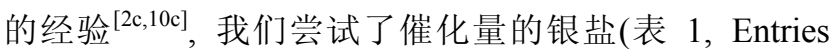
$12 \sim 13)$, 但是并没有得到满意的结果. 最终我们确定 了最优的反应条件为 2 equiv. 的 $\mathrm{AgNO}_{3}$ 为氧化剂, 反应 底物 2-异氧基-1,1'-联苯与二苯基磷氧的比例为 $1: 1.5$, 乙腈作为溶剂, 反应温度 $60{ }^{\circ} \mathrm{C}, \mathrm{Ar}$ 条件下反应 $2 \mathrm{~h}$.

表 1 磷自由基与 2-异氰基-1,1'-联苯(1a)的加成环化反应的条 件优化 ${ }^{a}$

Table 1 Optimization of conditions for the cyclization of the addition of P-radical to 2-isocyano-1,1'-biphenyl (1a)<smiles>C=C=Nc1ccccc1-c1ccccc1</smiles>

1a
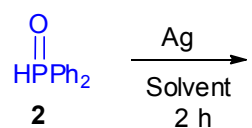

$2 \mathrm{~h}$

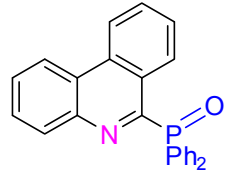

3a

\begin{tabular}{|c|c|c|c|c|c|}
\hline Entry & Oxidant (equiv.) & $1 a: 2$ & $T /{ }^{\circ} \mathrm{C}$ & Solvent & Yield ${ }^{b} / \%$ \\
\hline 1 & $\mathrm{AgNO}_{3}(1.2)$ & $1: 2.0$ & 30 & $\mathrm{MeCN}$ & 40 \\
\hline 2 & $\operatorname{AgOAc}(1.2)$ & $1: 2.0$ & 30 & $\mathrm{MeCN}$ & N.r \\
\hline 3 & $\mathrm{Ag}_{2} \mathrm{CO}_{3}(1.2)$ & $1: 2.0$ & 30 & $\mathrm{MeCN}$ & Trace \\
\hline 4 & $\mathrm{AgNO}_{3}(2)$ & $1: 2.0$ & 30 & $\mathrm{MeCN}$ & 65 \\
\hline 5 & $\mathrm{AgNO}_{3}(2)$ & $1: 2.0$ & 60 & $\mathrm{MeCN}$ & 83 \\
\hline 6 & $\mathrm{AgNO}_{3}(2)$ & $1: 2.0$ & 80 & $\mathrm{MeCN}$ & 82 \\
\hline 7 & $\mathrm{AgNO}_{3}(2)$ & $1: 2.0$ & 60 & DMF & 37 \\
\hline 8 & $\mathrm{AgNO}_{3}(2)$ & $1: 2.0$ & 60 & DCE & 69 \\
\hline 9 & $\mathrm{AgNO}_{3}(2)$ & $1: 2.0$ & 60 & Toluene & 74 \\
\hline 10 & $\mathrm{AgNO}_{3}(2)$ & $1: 1.2$ & 60 & $\mathrm{MeCN}$ & 78 \\
\hline 11 & $\mathrm{AgNO}_{3}(2)$ & $1: 1.5$ & 60 & $\mathrm{MeCN}$ & 86 \\
\hline 12 & $\mathrm{AgNO}_{3}(0.1)+\mathrm{K}_{2} \mathrm{~S}_{2} \mathrm{O}_{8}(1)$ & $1: 1.5$ & 60 & $\mathrm{MeCN}$ & 25 \\
\hline 13 & $\begin{array}{l}\mathrm{AgNO}_{3}(0.1)+\mathrm{Mg}\left(\mathrm{NO}_{3}\right)_{2} \bullet \\
6 \mathrm{H}_{2} \mathrm{O}(1)\end{array}$ & $1: 1.5$ & 60 & $\mathrm{MeCN}$ & 18 \\
\hline
\end{tabular}

${ }^{a}$ 反应条件: 1a $(0.2 \mathrm{mmol}), 2(0.3 \mathrm{mmol})$, 氧化剂 $(0.4 \mathrm{mmol})$, 溶剂为 $2 \mathrm{~mL}$, $\mathrm{Ar}$ 条件, 反应时间 $2 \mathrm{~h} ;^{b}$ 分离产率.

\section{2 底物范围的扩展}

基于上述优化的反应条件，我们对该反应底物范围 进行了考察(表 2，3a～31), 发现取代基的电子效应对反 应产率影响很大，当芳环上为给电子取代基时(表 $2,3 \mathbf{c}$ ), 能以较高产率得到产物, 而为拉电子取代基时(表 $2,3 \mathbf{d}$, 3e), 产率就会降低很多. 同时, 该反应能很好地兼容有
表 2 磷自由基与化合物 $\mathbf{1 a} \sim 11$ 的加成环化反应的底物范围 ${ }^{a}$

Table 2 Scope of the cyclization of the addition of P-radical to compounds $\mathbf{1 a} \sim 11$

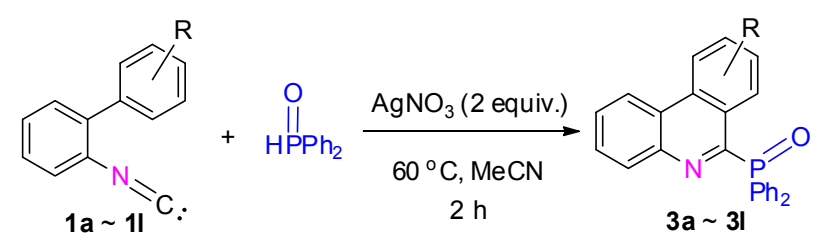

\begin{tabular}{lll}
\hline Compound & Product & Yield $^{b} / \%$ \\
\hline
\end{tabular}<smiles>[R]c1ccc2c(c1)c(P=O)nc1ccccc12</smiles>

3a

$3 \mathbf{b}$

$\mathrm{R}^{1}=\mathrm{H}$

86

$3 c$

$\mathrm{R}^{1}=\mathrm{Me}$

84

$\mathrm{R}^{1}=\mathrm{OMe}$

78

3d

$\mathrm{R}^{1}=\mathrm{CN}$

46

$3 e$

$\mathrm{R}^{1}=\mathrm{CO}_{2} \mathrm{Et}$

55

3f

$\mathrm{R}^{1}=\mathrm{Ph}$

82

$3 g$

$\mathrm{R}^{1}=\mathrm{Cl}$

77

$3 h$

$\mathrm{R}^{1}=\mathrm{F}$

81

$3 \mathbf{i}$<smiles>Cc1cc(Cl)c2c(c1)c([PH](=O)P)nc1ccccc12</smiles>

3j<smiles>O=C(c1ccccc1)c1c2ccccc2nc2ccccc12</smiles>

61<smiles>O=Pc1nc2ccccc2c2cc3c(cc12)OCO3</smiles>

3I<smiles></smiles>

厚应条件为: 1a $(0.2 \mathrm{mmol}), \mathbf{2}(0.3 \mathrm{mmol})$, 氧化剂 $(0.4 \mathrm{mmol})$, 溶剂为 2 $\mathrm{mL}, \mathrm{Ar}$ 条件，反应时间 $2 \mathrm{~h} ;{ }^{b}$ 分离产率.

卤素取代的底物(表 2,3g, 3h). 对于多取代的、更大共轭 芳环的和含有胡椒环的底物都能得到中等偏上的收率 (表 $2, \mathbf{3} \mathbf{i} \sim 3 \mathbf{k}$ ). 值得一提的是杂环底物也能得到目标产 物(表 2,31). 


\section{2 结论}

我们以 $\mathrm{AgNO}_{3}$ 作为氧化剂, 成功地实现了磷自由 基对异腈的加成环化反应, 为 $\mathrm{C}-\mathrm{P}$ 键的构建提供了一 种新的简单高效的方法. 并且合成了一系列 $\mathrm{N}-\mathrm{P}$ 配体 骨架. 该反应体系简单、条件温和、底物适用范围广泛 且产率高.

\section{3 实验部分}

\section{1 仪器与试剂}

实验所用溶剂使用前均按照处理溶剂的标准方法 进行. ${ }^{1} \mathrm{H}$ NMR, ${ }^{13} \mathrm{C}$ NMR, ${ }^{31} \mathrm{P}$ NMR, ${ }^{19} \mathrm{~F}$ NMR 均在德国 Bruker 公司 $400 \mathrm{MHz}$ 核磁共振仪上测定, 溶剂 $\mathrm{CDCl}_{3}$, 内标 TMS; 质谱数据的测定采用 Thermo Scientific DSQ II 质谱仪; 单晶数据测定使用 SuperNova, Dual, $\mathrm{Cu}$ at zero, Eos 衍射仪. 柱层析使用 200 300 目硅胶, 展开剂 为石油醚 $\left(60 \sim 90{ }^{\circ} \mathrm{C}\right)$ 和乙酸乙酯.

\section{2 实验方法}

在 $25 \mathrm{~mL}$ Schlenk 管中依次加入 $0.2 \mathrm{mmol}$ 的 2-异氧 基-1,1'-联苯, $0.3 \mathrm{mmol}$ 的二苯基磷氧, $0.4 \mathrm{mmol}$ 的 $\mathrm{AgNO}_{3}, 2 \mathrm{~mL}$ 乙腈. 使用双排管置换氩气三次, $60{ }^{\circ} \mathrm{C}$ 下 反应. TLC 检测反应, 反应结束后减压蒸馏除去溶剂, 柱层析, 得到目标产物 $\mathbf{3 a} \sim 31$.

菲啶-6-二苯基磷酰(3a): 产率 $86 \%$. ${ }^{1} \mathrm{H}$ NMR (400 $\left.\mathrm{MHz}, \mathrm{CDCl}_{3}\right) \delta: 9.51(\mathrm{~d}, J=8.3 \mathrm{~Hz}, 1 \mathrm{H}), 8.60$ (d, $J=8.4$ $\mathrm{Hz}, 1 \mathrm{H}), 8.55 \sim 8.51(\mathrm{~m}, 1 \mathrm{H}), 8.05 \sim 8.03(\mathrm{~m}, 1 \mathrm{H}), 7.97 \sim$ $7.92(\mathrm{~m}, 4 \mathrm{H}), 7.82 \sim 7.78(\mathrm{~m}, 1 \mathrm{H}), 7.70 \sim 7.64(\mathrm{~m}, 3 \mathrm{H})$, $7.51 \sim 7.41$ (m, $6 \mathrm{H}) ;{ }^{13} \mathrm{C}$ NMR $\left(100 \mathrm{MHz}, \mathrm{CDCl}_{3}\right) \delta: 156.7$ (d, $J=127.6 \mathrm{~Hz}), 142.6$ (d, $J=23.2 \mathrm{~Hz}), 133.3,132.4$ (d, $J=7.0 \mathrm{~Hz}), 132.2(\mathrm{~d}, J=9.3 \mathrm{~Hz}), 131.6(\mathrm{~d}, J=2.7 \mathrm{~Hz})$, 131.0, 130.9, 128.6 (d, $J=12.3 \mathrm{~Hz}), 128.4,128.1$ (d, $J=$ $12.0 \mathrm{~Hz}), 127.8,127.7,127.6,124.2$ (d, $J=2.6 \mathrm{~Hz}), 122.0$ (d, $J=3.3 \mathrm{~Hz}) ;{ }^{31} \mathrm{P}$ NMR (162 MHz, $\left.\mathrm{CDCl}_{3}\right) \delta: 28.31$; MS $m / z: 380(\mathrm{M}+1)^{+}$; HRMS calcd for $\mathrm{C}_{25} \mathrm{H}_{19} \mathrm{NOP}[\mathrm{M}+\mathrm{H}]^{+}$ 380.1204 , found 380.1187 .

8-甲基菲啶-6-二苯基磷酰(3b): 产率 84\%. ${ }^{1} \mathrm{H} \mathrm{NMR}$ $\left(400 \mathrm{MHz}, \mathrm{CDCl}_{3}\right) \delta: 9.33(\mathrm{~s}, 1 \mathrm{H}), 8.52 \sim 8.48(\mathrm{~m}, 2 \mathrm{H})$, $8.03 \sim 8.01(\mathrm{~m}, 1 \mathrm{H}), 7.97 \sim 7.92(\mathrm{~m}, 4 \mathrm{H}), 7.68 \sim 7.62(\mathrm{~m}$, $3 \mathrm{H}), 7.51 \sim 7.41(\mathrm{~m}, 6 \mathrm{H}), 2.54(\mathrm{~s}, 3 \mathrm{H}) ;{ }^{13} \mathrm{C}$ NMR $(100$ $\left.\mathrm{MHz}, \mathrm{CDCl}_{3}\right) \delta: 156.1$ (d, $\left.J=127.8 \mathrm{~Hz}\right), 142.3$ (d, $J=23.3$ Hz), 137.9, 133.0 (d, $J=104.1 \mathrm{~Hz}), 132.8,132.3$ (d, $J=9.0$ $\mathrm{Hz}), 131.5$ (d, $J=2.6 \mathrm{~Hz}), 130.9,130.4(\mathrm{~d}, J=6.8 \mathrm{~Hz})$, $128.1(\mathrm{~d}, J=101.9 \mathrm{~Hz}), 128.2,128.0(\mathrm{~d}, J=12.3 \mathrm{~Hz})$, $124.3(\mathrm{~d}, J=2.6 \mathrm{~Hz}), 121.9$ (d, $J=1.2 \mathrm{~Hz}), 121.8,21.9 ;{ }^{31} \mathrm{P}$ NMR (162 MHz, $\left.\mathrm{CDCl}_{3}\right) \delta: 28.08$; MS m/z: $394(\mathrm{M}+1)^{+}$;
HRMS calcd for $\mathrm{C}_{26} \mathrm{H}_{20} \mathrm{NOP}[\mathrm{M}+\mathrm{H}]^{+}$394.1355, found 394.1350 .

8-甲氧基菲啶-6-二苯基磷酰(3c)：产率 78\%. ${ }^{1} \mathrm{H}$ NMR (400 MHz, $\left.\mathrm{CDCl}_{3}\right) \delta: 9.02(\mathrm{~d}, J=2.6 \mathrm{~Hz}, 1 \mathrm{H})$, $8.55 \sim 8.48(\mathrm{~m}, 2 \mathrm{H}), 8.05 \sim 8.03(\mathrm{~m}, 1 \mathrm{H}), 7.99 \sim 7.94(\mathrm{~m}$, $4 \mathrm{H}), 7.70 \sim 7.62(\mathrm{~m}, 2 \mathrm{H}), 7.54 \sim 7.43(\mathrm{~m}, 7 \mathrm{H}), 3.94(\mathrm{~s}$, $3 \mathrm{H}) ;{ }^{13} \mathrm{C}$ NMR $\left(100 \mathrm{MHz}, \mathrm{CDCl}_{3}\right) \delta: 158.7,155.4(\mathrm{~d}, J=$ $128.4 \mathrm{~Hz}), 142.0$ (d, $J=23.1 \mathrm{~Hz}), 132.9$ (d, $J=104.0 \mathrm{~Hz}$ ), $132.2(\mathrm{~d}, J=9.2 \mathrm{~Hz}), 131.6(\mathrm{~d}, J=2.7 \mathrm{~Hz}), 131.0,129.4$ (d, $J=23.0 \mathrm{~Hz}), 128.8,128.1$ (d, $J=12.1 \mathrm{~Hz}), 127.6,127.0$ (d, $J=6.7 \mathrm{~Hz}), 124.5$ (d, $J=2.3 \mathrm{~Hz}), 123.6,122.6,121.6$, 107.4, 55.6; ${ }^{31} \mathrm{P}$ NMR $\left(162 \mathrm{MHz}, \mathrm{CDCl}_{3}\right) \delta$ : 27.60; MS $m / z: 410(\mathrm{M}+1)^{+}$; HRMS calcd for $\mathrm{C}_{26} \mathrm{H}_{20} \mathrm{NO}_{2} \mathrm{P}[\mathrm{M}+\mathrm{H}]^{+}$ 410.1304, found 410.1299.

8-氧基菲啶-6-二苯基磷酰(3d): 产率 46\%. ${ }^{1} \mathrm{H}$ NMR $\left(400 \mathrm{MHz}, \mathrm{CDCl}_{3}\right) \delta: 10.09(\mathrm{~d}, J=1.3 \mathrm{~Hz}, 1 \mathrm{H}), 8.72 \sim$ $8.69(\mathrm{~m}, 1 \mathrm{H}), 8.59 \sim 8.56(\mathrm{~m}, 1 \mathrm{H}), 8.14 \sim 8.11(\mathrm{~m}, 1 \mathrm{H})$, $8.00 \sim 7.94(\mathrm{~m}, 5 \mathrm{H}), 7.86 \sim 7.79(\mathrm{~m}, 2 \mathrm{H}), 7.58 \sim 7.54(\mathrm{~m}$, $2 \mathrm{H}), 7.50 \sim 7.46(\mathrm{~m}, 4 \mathrm{H}) ;{ }^{13} \mathrm{C} \mathrm{NMR}\left(100 \mathrm{MHz}, \mathrm{CDCl}_{3}\right) \delta$ : 156.5 (d, $J=126.0 \mathrm{~Hz}), 143.4(\mathrm{~d}, J=22.1 \mathrm{~Hz}), 134.9$ (d, $J=6.5 \mathrm{~Hz}), 133.9,132.1(\mathrm{~d}, J=104.9 \mathrm{~Hz}), 132.2(\mathrm{~d}, J=$ $9.5 \mathrm{~Hz}), 132.0$ (d, $J=2.8 \mathrm{~Hz}), 131.3,130.5,129.7,128.3$ $(\mathrm{d}, \quad J=12.3 \mathrm{~Hz}), 127.2(\mathrm{~d}, J=22.7 \mathrm{~Hz}), 123.4,122.9$, 122.6, 118.3, 111.4; ${ }^{31} \mathrm{P}$ NMR (162 MHz, $\left.\mathrm{CDCl}_{3}\right) \delta$ : 27.34; MS $m / z$ : $405(\mathrm{M}+1)^{+}$; HRMS calcd for $\mathrm{C}_{26} \mathrm{H}_{17} \mathrm{~N}_{2} \mathrm{OP}[\mathrm{M}+$ $\mathrm{H}]^{+}$405.1151, found 405.1147 .

8-甲酸乙酯基菲啶-6-二苯基磷酰(3e): 产率 55\%. ${ }^{1} \mathrm{H}$ NMR (400 MHz, $\left.\mathrm{CDCl}_{3}\right) \delta: 10.20(\mathrm{~d}, J=1.2 \mathrm{~Hz}, 1 \mathrm{H})$, $8.67 \sim 8.65(\mathrm{~m}, 1 \mathrm{H}), 8.59 \sim 8.57(\mathrm{~m}, 1 \mathrm{H}), 8.45 \sim 8.42(\mathrm{~m}$, $1 \mathrm{H}), 8.09 \sim 8.07(\mathrm{~m}, 1 \mathrm{H}), 8.02 \sim 7.97(\mathrm{~m}, 4 \mathrm{H}), 7.77 \sim 7.72$ (m, 2H), $7.55 \sim 7.44(\mathrm{~m}, 6 \mathrm{H}), 4.43(\mathrm{q}, J=7.1 \mathrm{~Hz}, 2 \mathrm{H})$, $1.43(\mathrm{t}, J=7.1 \mathrm{~Hz}, 3 \mathrm{H}) ;{ }^{13} \mathrm{C}$ NMR $\left(100 \mathrm{MHz}, \mathrm{CDCl}_{3}\right) \delta$ : 165.9, $157.6(\mathrm{~d}, J=126.5 \mathrm{~Hz}), 143.3(\mathrm{~d}, J=22.6 \mathrm{~Hz})$, $135.3(\mathrm{~d}, J=6.6 \mathrm{~Hz}), 132.6$ (d, $J=104.3 \mathrm{~Hz}), 132.2(\mathrm{~d}$, $J=9.2 \mathrm{~Hz}), 131.7(\mathrm{~d}, J=2.6 \mathrm{~Hz}), 131.1,130.8,130.5$, 129.7, 129.5, 129.1, 128.2 (d, $J=12.0 \mathrm{~Hz}), 127.0$ (d, $J=$ $22.8 \mathrm{~Hz}), 123.6(\mathrm{~d}, J=2.2 \mathrm{~Hz}), 122.5(\mathrm{~d}, J=19.3 \mathrm{~Hz})$, 61.3, 14.3; ${ }^{31} \mathrm{P}$ NMR (162 MHz, $\mathrm{CDCl}_{3}$ ) $\delta: 27.12$; MS $m / z$ : $452(\mathrm{M}+1)^{+}$; HRMS calcd for $\mathrm{C}_{28} \mathrm{H}_{22} \mathrm{NO}_{3} \mathrm{P}[\mathrm{M}+\mathrm{H}]^{+}$ 452.1410, found 452.1409 .

8-苯基菲啶-6-二苯基磷酰(3f): 产率 82\%. ${ }^{1} \mathrm{H}$ NMR $\left(400 \mathrm{MHz}, \mathrm{CDCl}_{3}\right) \delta: 9.82(\mathrm{~d}, J=1.6 \mathrm{~Hz}, 1 \mathrm{H}), 8.64 \sim 8.62$ $(\mathrm{m}, 1 \mathrm{H}), 8.54 \sim 8.51(\mathrm{~m}, 1 \mathrm{H}), 8.07 \sim 8.03(\mathrm{~m}, 2 \mathrm{H}), 8.01-$ $7.96(\mathrm{~m}, 4 \mathrm{H}), 7.70 \sim 7.66(\mathrm{~m}, 4 \mathrm{H}), 7.51 \sim 7.42(\mathrm{~m}, 8 \mathrm{H})$, $7.37 \sim 7.34(\mathrm{~m}, 1 \mathrm{H}) ;{ }^{13} \mathrm{C} \mathrm{NMR}\left(100 \mathrm{MHz}, \mathrm{CDCl}_{3}\right) \delta: 156.8$ 
(d, $J=127.3 \mathrm{~Hz}), 142.6$ (d, $J=23.2 \mathrm{~Hz}), 140.0$ (d, $J=43.1$ Hz), 133.0 (d, $J=104.0 \mathrm{~Hz}), 132.2$ (d, $J=9.0 \mathrm{~Hz}), 131.6$ $(\mathrm{d}, J=2.7 \mathrm{~Hz}), 131.5$ (d, $J=6.6 \mathrm{~Hz}), 131.0,129.9,128.9$, 128.7 (d, $J=25.2 \mathrm{~Hz}), 128.2$ (d, $J=22.9 \mathrm{~Hz}), 128.1,128.0$, $127.8,127.4,126.2,124.1$ (d, $J=2.3 \mathrm{~Hz}), 122.6,122.0 ;{ }^{31} \mathrm{P}$ NMR (162 MHz, $\left.\mathrm{CDCl}_{3}\right) \delta: 27.52 ; \mathrm{MS} \mathrm{m} / z: 456(\mathrm{M}+1)^{+}$; HRMS calcd for $\mathrm{C}_{31} \mathrm{H}_{22} \mathrm{NOP}[\mathrm{M}+\mathrm{H}]^{+}$456.1512, found 456.1517.

8-氯-菲啶-6-二苯基磷酰(3g): 产率 77\%, ${ }^{1} \mathrm{H}$ NMR $\left(400 \mathrm{MHz}, \mathrm{CDCl}_{3}\right) \delta: 9.66(\mathrm{~d}, J=2.1 \mathrm{~Hz}, 1 \mathrm{H}), 8.58 \sim 8.52$ $(\mathrm{m}, 2 \mathrm{H}), 8.08 \sim 8.05(\mathrm{~m}, 1 \mathrm{H}), 7.98 \sim 7.93(\mathrm{~m}, 4 \mathrm{H}), 7.79$ $(\mathrm{dd}, J=2.2,8.9 \mathrm{~Hz}, 1 \mathrm{H}), 7.76 \sim 7.71(\mathrm{~m}, 2 \mathrm{H}), 7.55 \sim 7.51$ $(\mathrm{m}, 2 \mathrm{H}), 7.48 \sim 7.44(\mathrm{~m}, 4 \mathrm{H}) ;{ }^{13} \mathrm{C}$ NMR $(100 \mathrm{MHz}$, $\left.\mathrm{CDCl}_{3}\right) \delta: 155.8(\mathrm{~d}, J=127.3 \mathrm{~Hz}), 142.6(\mathrm{~d}, J=22.6 \mathrm{~Hz})$, 133.5 (d, $J=78.1 \mathrm{~Hz}), 132.3$ (d, $J=9.3 \mathrm{~Hz}), 132.1,131.8$, 131.7, 131.1, 130.9 (d, $J=6.7 \mathrm{~Hz}), 129.1$ (d, $J=21.4 \mathrm{~Hz})$, 128.7 (d, $J=22.7 \mathrm{~Hz}), 128.2$ (d, $J=12.1 \mathrm{~Hz}), 127.6,123.7$, $121.9 ;{ }^{31} \mathrm{P}$ NMR (162 MHz, $\left.\mathrm{CDCl}_{3}\right) \delta: 27.35 ; \mathrm{MS} \mathrm{m} / z: 414$ $(\mathrm{M}+1)^{+}$; HRMS calcd for $\mathrm{C}_{25} \mathrm{H}_{17} \mathrm{CINOP}[\mathrm{M}+\mathrm{H}]^{+}$ 414.0809, found 414.0815.

8-氟-菲啶-6-二苯基磷酰(3h): 产率 $81 \%$. ${ }^{1} \mathrm{H}$ NMR $\left(400 \mathrm{MHz}, \mathrm{CDCl}_{3}\right) \delta: 9.33(\mathrm{dd}, J=2.6,10.2 \mathrm{~Hz}, 1 \mathrm{H})$, $8.62 \sim 8.59(\mathrm{~m}, 1 \mathrm{H}), 8.50 \sim 8.48(\mathrm{~m}, 1 \mathrm{H}), 8.07 \sim 8.05(\mathrm{~m}$, $1 \mathrm{H}), 7.98 \sim 7.93(\mathrm{~m}, 4 \mathrm{H}), 7.73 \sim 7.66(\mathrm{~m}, 2 \mathrm{H}), 7.59 \sim 7.43$ $(\mathrm{m}, 7 \mathrm{H}) ;{ }^{13} \mathrm{C} \mathrm{NMR}\left(100 \mathrm{MHz}, \mathrm{CDCl}_{3}\right) \delta: 161.2(\mathrm{~d}, J=$ $247.8 \mathrm{~Hz}$ ), 155.8 (dd, $J=4.3,127.7 \mathrm{~Hz}), 142.3$ (d, $J=22.6$ $\mathrm{Hz}), 132.6$ (d, $J=104.3 \mathrm{~Hz}), 132.2$ (d, $J=9.0 \mathrm{~Hz}), 131.8$ (d, $J=2.7 \mathrm{~Hz}), 131.1,128.8(\mathrm{~d}, J=62.7 \mathrm{~Hz}), 128.2$ (d, $J=$ $12.2 \mathrm{~Hz}), 124.5$ (d, $J=8.7 \mathrm{~Hz}), 123.8,121.8,120.4$ (d, $J=$ $24.3 \mathrm{~Hz}), 113.1(\mathrm{~d}, J=23.1 \mathrm{~Hz}) ;{ }^{31} \mathrm{P}$ NMR $(162 \mathrm{MHz}$, $\left.\mathrm{CDCl}_{3}\right) \quad \delta: 27.44 ;{ }^{19} \mathrm{~F}$ NMR $\left(376 \mathrm{MHz}, \mathrm{CDCl}_{3}\right) \quad \delta$ : - 109.58; MS m/z: $398(\mathrm{M}+1)^{+}$; HRMS calcd for $\mathrm{C}_{25} \mathrm{H}_{17} \mathrm{FNOP}[\mathrm{M}+\mathrm{H}]^{+}$398.1105, found 398.1103.

10-氯-8-甲基菲啶-6-二苯基磷酰(3i)：产率 $67 \%$. ${ }^{1} \mathrm{H}$ NMR (400 MHz, $\left.\mathrm{CDCl}_{3}\right) \delta: 9.80 \sim 9.76(\mathrm{~m}, 1 \mathrm{H}), 9.41(\mathrm{~s}$, $1 \mathrm{H}), 8.04 \sim 8.01(\mathrm{~m}, 1 \mathrm{H}), 7.93 \sim 7.88(\mathrm{~m}, 4 \mathrm{H}), 7.76(\mathrm{~d}, J=$ $1.4 \mathrm{~Hz}, 1 \mathrm{H}), 7.72 \sim 7.68(\mathrm{~m}, 2 \mathrm{H}), 7.54 \sim 7.50(\mathrm{~m}, 2 \mathrm{H})$, $7.47 \sim 7.43(\mathrm{~m}, 4 \mathrm{H}), 2.52(\mathrm{~s}, 3 \mathrm{H}) ;{ }^{13} \mathrm{C}$ NMR $(100 \mathrm{MHz}$, $\left.\mathrm{CDCl}_{3}\right) \delta: 156.1(\mathrm{~d}, J=127.6 \mathrm{~Hz}), 143.3(\mathrm{~d}, J=23.1 \mathrm{~Hz})$, $138.3,136.2,132.8$ (d, $J=105.0 \mathrm{~Hz}), 132.2$ (d, $J=9.0 \mathrm{~Hz})$, $131.7(\mathrm{~d}, J=2.7 \mathrm{~Hz}), 131.2,130.8$ (d, $J=3.1 \mathrm{~Hz}), 130.4$, 130.1, $128.4(\mathrm{~d}, J=25.5 \mathrm{~Hz}), 128.2,128.1,127.5,127.2$ $(\mathrm{d}, J=6.7 \mathrm{~Hz}), 126.1,123.4(\mathrm{~d}, J=2.5 \mathrm{~Hz}), 21.3 ;{ }^{31} \mathrm{P}$ NMR (162 MHz, $\left.\mathrm{CDCl}_{3}\right) \delta: 29.04 ; \mathrm{MS} \mathrm{m} / z: 428(\mathrm{M}+1)^{+}$; HRMS calcd for $\mathrm{C}_{26} \mathrm{H}_{19} \mathrm{CINOP}[\mathrm{M}+\mathrm{H}]^{+}$428.0966, found
428.0961 .

苯并 $[i]$ 菲啶-5-二苯基磷酰(3j): 产率 $61 \%$. ${ }^{1} \mathrm{H}$ NMR $\left(400 \mathrm{MHz}, \mathrm{CDCl}_{3}\right) \delta: 9.59$ (d, $\left.J=8.1 \mathrm{~Hz}, 1 \mathrm{H}\right), 8.63 \sim 8.57$ $(\mathrm{m}, 2 \mathrm{H}), 8.13(\mathrm{~d}, J=8.9 \mathrm{~Hz}, 1 \mathrm{H}), 7.95 \sim 7.93(\mathrm{~m}, 1 \mathrm{H})$, $7.87 \sim 7.79(\mathrm{~m}, 5 \mathrm{H}), 7.75 \sim 7.68(\mathrm{~m}, 2 \mathrm{H}), 7.55 \sim 7.48(\mathrm{~m}$, $2 \mathrm{H}), 7.44 \sim 7.40(\mathrm{~m}, 2 \mathrm{H}), 7.37 \sim 7.33(\mathrm{~m}, 4 \mathrm{H}) ;{ }^{13} \mathrm{C} \mathrm{NMR}$ $\left(100 \mathrm{MHz}, \mathrm{CDCl}_{3}\right) \delta: 154.9$ (d, $\left.J=128.4 \mathrm{~Hz}\right), 142.8$ (d, $J=22.9 \mathrm{~Hz}), 134.5,133.5,133.3$ (d, $J=6.6 \mathrm{~Hz}), 132.7$, 132.5, $132.0(\mathrm{~d}, J=8.9 \mathrm{~Hz}), 131.1(\mathrm{~d}, J=2.7 \mathrm{~Hz}), 130.4$, $130.2,128.9$ (d, $J=11.6 \mathrm{~Hz}), 128.8,127.9$ (d, $J=12.0 \mathrm{~Hz})$, 127.4 (d, $J=28.4 \mathrm{~Hz}), 126.5,125.4$ (d, $J=22.6 \mathrm{~Hz}), 123.8$ (d, $J=2.7 \mathrm{~Hz}), 122.5,119.5(\mathrm{~d}, J=1.9 \mathrm{~Hz}) ;{ }^{31} \mathrm{P}$ NMR $(162$ $\left.\mathrm{MHz}, \mathrm{CDCl}_{3}\right) \delta: 33.53$; MS m/z: $430(\mathrm{M}+1)^{+}$; HRMS calcd for $\mathrm{C}_{29} \mathrm{H}_{20} \mathrm{NOP}[\mathrm{M}+\mathrm{H}]^{+}$430.1355, found 430.1350 .

$[1,3]$ 二氧杂环戊烯并 $[4,5-j]$ 菲啶-6-二苯基磷酰(3k): 产率 $71 \%$. ${ }^{1} \mathrm{H}$ NMR $\left(400 \mathrm{MHz}, \mathrm{CDCl}_{3}\right) \delta: 9.01(\mathrm{~s}, 1 \mathrm{H})$, $8.34 \sim 8.32(\mathrm{~m}, 1 \mathrm{H}), 8.00 \sim 7.92(\mathrm{~m}, 5 \mathrm{H}), 7.88(\mathrm{~s}, 1 \mathrm{H})$, $7.64 \sim 7.61(\mathrm{~m}, 2 \mathrm{H}), 7.52 \sim 7.41(\mathrm{~m}, 6 \mathrm{H}), 6.09(\mathrm{~s}, 2 \mathrm{H}) ;{ }^{13} \mathrm{C}$ NMR (100 MHz, $\left.\mathrm{CDCl}_{3}\right) \delta: 154.5(\mathrm{~d}, J=129.0 \mathrm{~Hz}), 151.2$, 148.2, 142.4 (d, $J=23.3 \mathrm{~Hz}), 133.0$ (d, $J=104.2 \mathrm{~Hz})$, $132.3(\mathrm{~d}, J=9.3 \mathrm{~Hz}), 131.6$ (d, $J=2.7 \mathrm{~Hz}), 130.9,130.7$ (d, $J=7.3 \mathrm{~Hz}), 128.1(\mathrm{~d}, J=29.3 \mathrm{~Hz}), 128.1$ (d, $J=11.9$ $\mathrm{Hz}), 124.9$ (d, $J=23.5 \mathrm{~Hz}), 124.3$ (d, $J=2.6 \mathrm{~Hz}), 121.9$, 105.5, 102.0, 99.8 (d, $J=1.3 \mathrm{~Hz}) ;{ }^{31} \mathrm{P} \mathrm{NMR}(162 \mathrm{MHz}$, $\left.\mathrm{CDCl}_{3}\right) \delta: 28.15$; MS m/z: $424(\mathrm{M}+1)^{+}$; HRMS calcd for $\mathrm{C}_{26} \mathrm{H}_{18} \mathrm{NO}_{3} \mathrm{P}[\mathrm{M}+\mathrm{H}]^{+}$424.1097, found 424.1102.

噻吩并[3,2-c]喹啉-4-二苯基磷酰(3I): 产率 $20 \% .{ }^{1} \mathrm{H}$ NMR (400 MHz, $\left.\mathrm{CDCl}_{3}\right) \delta: 8.67(\mathrm{~d}, J=5.6 \mathrm{~Hz}, 1 \mathrm{H})$, $8.19 \sim 8.14(\mathrm{~m}, 2 \mathrm{H}), 8.03 \sim 7.98(\mathrm{~m}, 4 \mathrm{H}), 7.72 \sim 7.65(\mathrm{~m}$, $4 \mathrm{H}), 7.60(\mathrm{~d}, J=5.44 \mathrm{~Hz}, 1 \mathrm{H}), 7.54 \sim 7.50(\mathrm{~m}, 2 \mathrm{H}), 7.48 \sim$ $7.43(\mathrm{~m}, 4 \mathrm{H}) ;{ }^{13} \mathrm{C}$ NMR $\left(100 \mathrm{MHz}, \mathrm{CDCl}_{3}\right) \delta: 152.3(\mathrm{~d}$, $J=129.7 \mathrm{~Hz}), 146.4(\mathrm{~d}, J=9.2 \mathrm{~Hz}), 142.8(\mathrm{~d}, J=21.9 \mathrm{~Hz})$, 135.6, 135.3, 132.6 (d, $J=103.5 \mathrm{~Hz}), 132.3$ (d, $J=9.1 \mathrm{~Hz})$, 131.7 (d, $J=2.8 \mathrm{~Hz}), 131.1,128.6$ (d, $J=2.7 \mathrm{~Hz}), 128.3$, 128.1, 126.6, 125.4, 124.4 (d, $J=2.2 \mathrm{~Hz}), 123.4 ;{ }^{31} \mathrm{P}$ NMR $\left(162 \mathrm{MHz}, \mathrm{CDCl}_{3}\right) \delta: 24.20$; $\mathrm{MS} \mathrm{m} / z: 386(\mathrm{M}+1)^{+}$; HRMS calcd for $\mathrm{C}_{23} \mathrm{H}_{16} \mathrm{NOPS}[\mathrm{M}+\mathrm{H}]^{+} 386.0763$, found 386.0768 .

辅助材料(Supporting Information) 产物 3a $\sim 31$ 的核 磁表征谱图和化合物 $\mathbf{3 a}$ 的晶体结构数据. 这些材料可 以免费从本刊网站(http://sioc-journal. cn/)上下载. 


\section{References}

[1] (a) Bialy, L.; Waldmann, H. Angew. Chem., Int. Ed. 2005, 44, 3814.

(b) Albrecht, L.; Albrecht, A.; Krawczyk, H.; Jorgensen, K. A. Chem.-Eur. J. 2010, 16, 28.

(c) George, A.; Veis, A. Chem. Rev. 2008, 108, 4670.

(d) Jeught, S. V.; Stevens, C. V. Chem. Rev. 2009, 109, 2672.

(e) Zhao, D.; Wang, R. Chem. Soc. Rev. 2012, 41, 2095.

[2] (a) Yang, B.; Yang, T.-T.; Li, X. A.; Wang, J. J.; Yang, S.-D. Org. Lett. 2013, 15, 5024.

(b) Hu, J.; Zhao, N.; Yang, B.; Wang, G.; Guo, L.-N.; Liang, Y. M.; Yang, S.-D. Chem.-Eur. J. 2011, 17, 5516.

(c) Li, Y.-M.; Sun, M.; Wang, H.-L.; Tian, Q.-P.; Yang, S.-D. Angew. Chem., Int. Ed. 2013, 52, 3972.

(d) Sun, M.; Zhang, H.-Y.; Han, Q.; Yang, K.; Yang, S.-D. Chem.-Eur. J. 2011, 17, 9566.

(e) Zhang, H.-Y.; Sun, M.; Ma, Y.-N.; Tian, Q.-P.; Yang, S.-D. Org. Biomol. Chem. 2012, 10, 9627.

[3] (a) Kalek, M.; Ziadi, A.; Stawinski, J. Org. Lett. 2008, 10, 4637.

(b) Zhang, X. H.; Liu, H. Z.; Hu, X. M.; Tang, G.; Zhu, J.; Zhao, Y. F. Org. Lett. 2011, 13, 3478 .

(c) Bruch, A.; Fukazawa, A.; Yamaguchi, E.; Yamaguchi, S.; Studer, A. Angew. Chem., Int. Ed. 2011, 50, 12094.

[4] Hirao, T.; Masunaga, T.; Ohshiro, Y.; Agawa, T. Synthesis 1981, 56.
[5] Petrakis, K. S.; Nagabhushan, T. L. J. Am. Chem. Soc. 1987, 109, 2831.

[6] Luo, Y.; Wu, J. Organometallics 2009, 28, 6823.

[7] (a) Hu, G.; Chen, W.; Fu, T.; Peng, Z.; Qiao, H.; Cao, Y.; Zhao, Y. F. Org. Lett. 2013, 15, 5362.

(b) Zhuang, R.; Xu, J.; Cai, Z.; Tang, G.; Fang, M.; Zhao, Y. F. Org. Lett. 2011, 13, 2110.

[8] (a) Pan, X.-Q.; Zou, J.-P.; Zhang, G.-L.; Zhang, W. Chem. Commun. 2010, 46, 1721.

(b) Zhou, J.; Zhang, G.-L.; Zou, J.-P.; Zhang, W. Eur. J. Org. Chem. 2011, 3412.

(c) Pan, X.-Q.; Wang, L.; Zou, J.-P.; Zhang, W. Chem. Commun. 2011, 47, 7875.

(d) Chen, Y.-R.; Duan, W.-L. J. Am. Chem. Soc. 2013, 135, 16754.

(e) Tayama, O.; Nakano, A.; Iwahama, T.; Sakaguchi, S.; Ishii, Y. J. Org. Chem. 2004, 69, 5494.

(e) Snider, B. B. Chem. Rev. 1996, 96, 339.

[9] Zhang, B.; Daniliuc, C. G.; Studer, A. Org. Lett. 2014, 16, 250.

[10] (a) Xiang, C.-B.; Bian, Y.-J.; Mao, X.-R.; Huang, Z.-Z. J. Org. Chem. 2012, 7706.

(b) Wang, H.; Guo, L.-N.; Duan, X.-H. Chem. Commun. 2013, 49, 10370.

(c) Wei, X.-H.; Li, Y.-M.; Zhou, A.-X.; Yang, T.-T.; Yang, S.-D. Org. Lett. 2013, 15, 4158.

(d) Yuan, Y.-Z.; Shen, T.; Wang, K.; Jiao, N. Chem.-Asian J. 2013, 8, 2932. 